\title{
Repensando la Agresión entre Adolescentes: Una Revisión de la Teoría de Estrategias de Control de Recursos en Latinoamérica
}

\author{
Rethinking Aggression Among Adolescents: A Review of Resource \\ Control Strategies Theory in Latin America
}

\section{Eduardo Franco Chalco ${ }^{1}$}

\section{Revista}

\section{Educación y Sociedad}

\section{Citar como: Franco, E. (2021).} Repensando la Agresión entre Adolescentes: Una Revisión de la Teoría de Estrategias de Control de Recursos en Latinoamérica. Revista Educación y Sociedad, 02(03), 2-7. doi: $10.53940 /$ reys.v2i3.61

Artículo recibido: 20/03/2021 Artículo aprobado: 20/05/2021 Arbitrado por pares

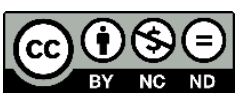

\section{ACEES}

\section{Resumen}

La Teoría de Control de Recursos es un planteamiento con un poco más de 20 años en la literatura de la psicología del desarrollo. Esta sugiere que los seres humanos desplegamos estrategias coercitivas y/o estrategias prosociales para alcanzar nuestros objetivos sociales. Este marco conceptual ha logrado identificar que la conducta agresiva entre adolescentes es utilizada instrumentalmente para obtener estatus social. Sin embargo, estos estudios son escasos con poblaciones latinoamericanas, por lo que es necesario promoverlos para diseñar intervenciones contextualizadas para la agresión escolar.

\section{Palabras clave: agresión, prosocialidad, adolescentes, escuelas}

\section{Abstract}

The Resource Control Theory is a proposal with more than 20 years in the developmental psychology literature. This theory proposes that humans display coercive and prosocial strategies in order to attain our social goals. Under this framework, aggressive behavior has been identified as an instrument for adolescents to attain social status. However, Latin-American studies are scares. This type of studies must be promoted in order to be able to design contextualized interventions for school aggression.

Key words: aggression, prosociality, adolescents, schools 


\section{Introducción}

La conducta agresiva entre adolescentes se considera un serio problema en las escuelas alrededor del mundo. No solo porque esta se encuentre asociada a fenómenos complejos como el bullying o acoso escolar (Hawley, Little, y Rodkin, 2007). Sino porque tiene efectos negativos en el desarrollo y la salud mental tanto en las víctimas (Graham, Bellmore y Jovonen, 2003; Schwartz, Lansford, Dodge, Pettit y Bates, 2015; Zeman, Shipman y Suveg, 2002) como en los agresores (Campbell, Spieker, Vandergrift, Belsky y Burcinal, 2010; Cleverley, Szatamari, Vallacourt, Boyle y Lipmann, 2012). Múltiples enfoques han intentado explicar el origen de la conducta agresiva, la mayoría de los enfoques consideran a la agresión como una conducta desadaptativa que resulta del mal procesamiento de la información social del contexto donde se desarrollan los adolescentes (Coie y Dodge, 1994).

Sin embargo, un enfoque relativamente reciente cambió la pregunta de ¿por qué un adolescente es agresivo?, a ¿para qué un adolescente es agresivo? Patricia Hawley (1999) planteó que las conductas agresivas forman parte de un set de estrategias que permiten a los individuos conseguir recursos sociales y materiales, por lo que la agresión desde esta perspectiva es considerada una herramienta social. Además, Hawley (2003) identifica un perfil específico de adolescentes que también utiliza estrategias prosociales en combinación con la agresión que resulta útil para mejorar su estatus social.

En el desarrollo del presente artículo se presentarán detalles de la Teoría de Control de Recursos (TCR, Hawley, 1999); además, se presentará evidencia actualizada que permite hipotetizar sobre si este fenómeno se desarrolla también en las escuelas peruanas. Para de este modo concluir sobre la necesidad de desarrollar programas de investigación que identifiquen perfiles de estudiantes que mejoren las intervenciones en agresión escolar en nuestro país.

\section{Teoría de Control de Recursos}

Esta teoría postulada en 1999 por Patricia Hawley, tiene una perspectiva evolucionista y plantea que los seres humanos como especie tenemos disposiciones que nos impulsan a conseguir dominación social, debido a que los individuos con estatus prominentes en los grupos tienen un mayor acceso a recursos que permiten su supervivencia y reproducción. Desde esta perspectiva teórica la dominación social se define como la capacidad de maximizar el control de recursos materiales y sociales (Hawley, 2003). En este sentido, Hawley (2003) propone que se van poniendo en práctica, desde tempranas etapas del desarrollo, múltiples estrategias para ser dominante, entre las cuales se definen dos formas principales: estrategias prosociales y estrategias coercitivas.

Se entiende por estrategia prosocial como una serie de conductas aceptadas socialmente que permiten obtener recursos en interacción con otras personas; en este sentido, se encuentran conductas de cooperación, reciprocidad, y/o formación de alianzas (Franco y Berger, 2021; Hawley, 2007). Por el contrario, las estrategias coercitivas son conductas como la intimidación, manipulación, agresión que son utilizadas de manera intencional para obtener recursos, en ese sentido se puede mencionar que las estrategias coercitivas son utilizadas para forzar a otros a cumplir la voluntad del individuo que las realiza (Franco y Berger, 2021; Hawley, 2007).

A primera vista se puede considerar que las estrategias coercitivas y las estrategias prosociales son fenómenos opuestos, sin embargo, ambas comparten un mismo fin por lo que algunos sujetos pueden utilizar ambas estrategias para maximizar el control de recursos. Esto se relaciona con la hipótesis de centralidad social de la TCR, que propone que aquellos individuos que combinan ambos tipos de estrategias en diversas situaciones alcanzan los niveles más altos de dominación social (Hawley, 1999). 
Desde esta hipótesis es que se toma una perspectiva centrada en la persona, que permite identificar perfiles de individuos según la frecuencia de uso de las estrategias de control de recursos.

\section{Enfoque centrado en la persona}

Hawley (2003) propuso la identificación de 5 grupos de personas según la frecuencia del uso de cierta forma de estrategia de control de recursos, con cortes arbitrarios en los percentiles de cada una de las dimensiones de control coercitivo y prosocial. En la Figura 1 se pueden observar los puntos donde se realizan los cortes de percentiles y los nombres de los 5 perfiles identificados. A partir de estos cortes que se identifican los controladores biestratégicos (superior al percentil 66 en ambas estrategias). También se identifican controladores prosociales (superior al percentil 66 en estrategias prosociales, e inferior al percentil 66 en estrategias coercitivas), controladores coercitivos (superior al percentil 66 en estrategias coercitivas, e inferior al percentil 66 en estrategias prosociales), controladores típicos (uso promedio de ambas estrategias) y no controladores (inferior al percentil 33 en ambas estrategias).

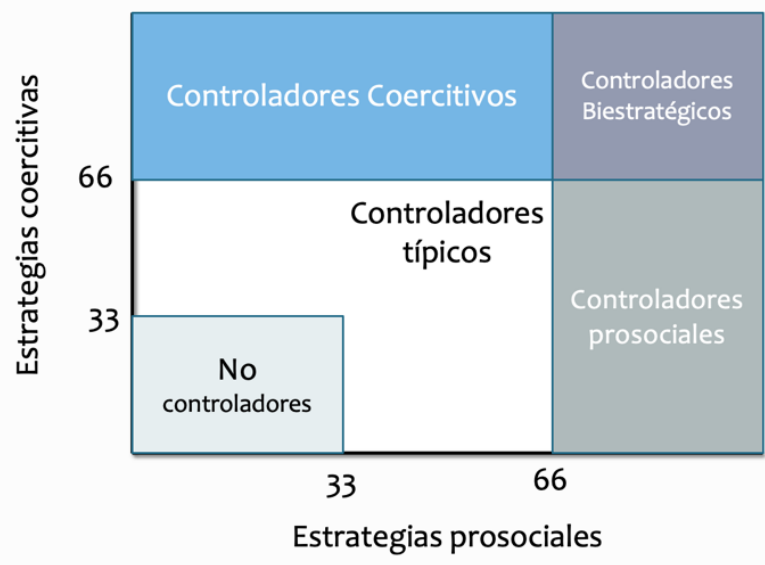

Figura 1. Cortes de percentiles para los perfiles de estrategias de control de recursos.

Algunos de los resultados que ha permitido encontrar esta perspectiva son que los controladores biestratégicos tienden a ser los estudiantes más populares en su grupo de pares, mientras que los no controladores tienden a ser los menos populares. Por otro lado, los controladores prosociales no son tan populares como los controladores biestratégicos, pero si tienen altos niveles de aceptación social (Hawley, 2003). Del mismo modo, también se ha identificado que los controladores biestratégicos utilizan la agresión y el bullying como instrumentos para obtener dominación en su grupo de pares (Olthof, Goosens, Vermade, Aleva, y van der Meulen, 2011). Mientras que, los no controladores tienden a mayor retraimiento social que el resto de sus compañeros (Findley y Ojanen, 2013). Algunos estudios han Ilamado a los controladores biestratégicos como "Maquiavélicos" debido a que utilizan todos los medios que están en sus manos para alcanzar sus metas sociales (Hawley, 2003).

\section{Evidencia latinoamericana de la TCR}

La importancia de esta perspectiva está en que muestra que la conducta agresiva y otra serie de estrategias coercitivas son útiles en ciertos contextos. Es más, se puede observar que las estrategias coercitivas se vuelven normativas en ciertos espacios, por lo que permiten que los adolescentes que las 
utilizan alcancen altos niveles de estatus social entre sus compañeros. Lamentablemente, y a pesar de que la TCR tiene más de 20 años en la literatura de la psicología del desarrollo son escasos los estudios que han intentado comprobar esta teoría en población latinoamericana.

Berger, Batanova y Cance (2015) ensayaron una aproximación a la identificación de un perfil "Maquiavélico" entre preadolescentes chilenos. En este estudio se pudo identificar un perfil de estudiantes agresivos-populares y un perfil de estudiantes prosociales-preferidos socialmente, más no se encontró un perfil de estudiantes que combinen ambas conductas. Una limitación de este estudio fue que utilizaron como una aproximación de las estrategias de control de recursos a la conducta agresiva y la conducta prosocial específicamente. Sin embargo, la agresión y la prosocialidad pueden ser también reacitvas, en otras palabras, en respuesta a que alguien más fue agresivo o prosocial con ellos (Findleyvan Nostrand y Ojanen, 2018; Prinstein y Cillessen, 2003). Sin embargo, las estrategias de control de recursos son siempre proactivas o intencionales (Hawley, 1999). Esto puede observarse en los consistentes resultados de investigaciones que encuentran una correlación positiva entre las estrategias coercitivas y prosociales (Hawley, 2007) y la correlación negativa o nula entre la conducta agresiva y prosocial (Cillessen, Mayeux, Ha, de Bruyn, y LaFontana, 2014). Esta limitación se dio también ante la ausencia de un instrumento que permita medir directamente las estrategias de control de recursos en español. Este vació en la literatura fue abordado posteriormente por Franco y Berger (2021) que tradujeron y validaron el Inventario de Estrategias de Control de Recursos (IECR, Hawley, 2007) para una población de preadolescentes chilenos.

El estudio de Berger y colaboradores (2015) abordó también otra crítica común a la TCR, la cual indica que al utilizar cortes arbitrarios en percentiles de dos dimensiones de la escala se estaría forzando la aparición de los 5 perfiles de estrategias de control de recursos. Por lo que en dicho estudio se utilizó un modelo de Análisis de Clases Latentes que permite identificar subpoblaciones de individuos basadas en ciertas características conductuales. Debido a la limitación mencionada previamente, los grupos hallados no necesariamente confirman o confrontan lo postulado por Hawley. Esta limitación fue abordada posteriormente por Franco (2020) donde se utilizó el IECR para evaluar un grupo de 630 preadolescentes chilenos, y además se utilizó un Análisis de Clases Latentes para identificar los perfiles. Específicamente se encontraron 3 perfiles que cumplían con las características de los grupos de controladores biestratégicos, controladores prosociales y no controladores.

Con respecto al apoyo a la hipótesis de centralidad social se pudo identificar que los controladores biestratégicos son los más populares dentro de sus grupos de pares y que son tan aceptados como los controladores prosociales. Los no controladores tenían niveles de popularidad similares a los de los controladores prosociales, sin embargo, eran rechazados por sus pares. Este estudio también investigó por nominación de pares cuales eran las conductas más utilizadas por los estudiantes que fueron clasificados en los tres perfiles. Se encontró que los controladores biestratégicos y los no controladores eran considerados como los estudiantes más agresivos en sus entornos.

Los resultados del estudio de Franco (2020) iluminan varios puntos a considerar sobre la TCR en una población latinoamericana. Primeramente, es necesario diferenciar si el hecho de que no se encontraran los perfiles de controladores típicos y controladores coercitivos se debe a una diferencia con las poblaciones anglosajonas con las que se desarrolló la teoría o a las diferencias metodológicas para la clasificación. Sin embargo, es muy llamativo observar que los estudiantes chilenos entregaron un estatus prominente a aquellos estudiantes que combinaban estrategias coercitivas y estrategias prosociales, y rechazaron a estudiantes que solo usaban estrategias coercitivas. Si bien esto coincide con lo postulado por la teoría, nos sugiere que en poblaciones latinoamericanas hay un grupo de adolescentes a los que se les permite comportarse de manera agresiva, y que son admirados por sus pares. 
Este último punto es importante debido a que los programas de intervención contra el bullying y la agresión escolar se concentran por lo general en castigar las conductas agresivas, sin cuestionarse ¿Qué ganan los estudiantes al comportarse de ese modo? Es posible que también en el Perú la agresión sea una estrategia útil para alcanzar altos niveles de popularidad. Esto es especialmente relevante durante la preadolescencia, donde el conseguir popularidad es el objetivo social más predominante (Cillessen et al., 2014). Sin embrago, la utilidad de la agresión es una hipótesis que necesita ser confirmada con evidencia empírica, para de este modo promover programas de intervención orientados a modificar los símbolos de estatus entre los adolescentes en el Perú.

\section{Conclusiones}

La Teoría de Control de Recursos (Hawley, 1999) es una perspectiva relativamente novedosa en la literatura de la psicología del desarrollo en Latinoamérica. A pesar de sus aportes para la comprensión de la conducta social de los adolescentes en las escuelas, esta no ha sido estudiada a profundidad en estudios transculturales que permitan confirmar las hipótesis presentadas por la teoría hace más de 20 años. Además, se resalta la necesidad de plantear estudios longitudinales que permitan conocer las trayectorias de desarrollo de los controladores biestratégicos. Por otro lado, la importancia de promover estudios en esta línea de investigación está en que ayuda a diseñar intervenciones enfocadas a cambiar aspectos específicos del contexto de pares de los adolescentes, identificando sus perfiles conductuales y los roles que juegan en sus ecosistemas. Y de este modo mejorar la calidad de vida y la experiencia escolar de los estudiantes peruanos.

\section{Referencias}

Berger, C., Batanova, M., y Cance, J. D. (2015). Aggressive and Prosocial? Examining Latent Profiles of Behavior, Social Status, Machiavellianism, and Empathy. Journal of Youth and Adolescence, 44(12), 2230-2244. doi: 10.1007/s10964-015-0298-9

Campbell, S. B., Spieker, S., Vandergrift, N., Belsky, J., y Burchinal, M. (2010). Predictors and sequaele of trajectories of physical aggression in school-age boys and girls. Development and Psychopathology, 22, 133-150. doi: 10.1017/s0954579409990319

Cillessen, A. H. N., Mayeux, L., Ha, T., de Bruyn, E. H., y LaFontana, K. M. (2013). Aggressive effects of prioritizing popularity in early adolescence. Aggressive Behavior, 40(3), 204-213. doi: 10.1002/ab.21518

Cleverley, K., Szatmari, P., Vaillancourt, T., Boyle, M. y Lipman, E. (2012). Developmental trajectories of physical and indirect aggression from late childhood to adolescence: sex differences and outcomes in emerging adulthood. Journal of the American Academy of Child \& Adolescent Psychiatry, 51(10), 1037-1051. doi: 10.1016/j.jaac.2012.07.010

Crick, N. R., y Dodge, K. A. (1994). A review and reformulation of the social-information processing mechanism in children's social adjustments. Psychological Bulletin, 115(1), 74-101. doi: 10.1037/00332909.115.1.74

Findley-Van Nostrand, D., y Ojanen, T. (2018). Forms of Prosocial Behaviors are Differentially Linked to Social Goals and Peer Status in Adolescents. The Journal of Genetic Psychology, 179(6), 329-342. doi: 10.1080/00221325.2018.1518894

Findley, D., y Ojanen, T. (2013). Adolescent resource control: Associations with physical and relational aggression, prosocial and withdrawn behaviors, and peer regard. International Journal of Behavioral Development, 37(6), 518-529. doi: 10.1177/0165025413503420

Franco, E. (2020). Strategies for Status Attainment in Peer Early Adolescent Contexts (Tesis Doctoral). Pontificia Universidad Católica de Chile. Santiago, Chile. Recuperado de https://repositorio.uc.cl/xmlui/bitstream/handle/11534/36923/PhDThesis_v1.1.pdf 
Franco, E., y Berger, C. (2021). Inventario de Estrategias de Control de Recursos: Adaptación en Preadolescentes Chilenos. Revista de Psicología de la Pontificia Universidad Católica del Perú, 39(1), 60-83. doi: $10.18800 /$ psico.202101.003

Graham, S., Bellmore, A. y Juvonen, J. (2003). Peer victimization in middle school: When self and peer views diverge. Journal of Applied School Psychology, 19(2), 117-137. doi: 10.1300/Joo8v19no2_08

Hawley, P. H. (1999). The Ontogenesis of Social Dominance: A Strategy-Based Evolutionary Perspective. Developmental Review, 19(1), 97-132. doi: 10.1006/drev.1998.0470

Hawley, P. H. (2003). Prosocial and coercive configurations of resource control in early adolescence: A case for the well-adapted Machiavellian. Merril-Palmer Quarterly, 49(3), 279309. doi: $10.1353 / \mathrm{mpq} \cdot 2003.0013$

Hawley, P. H. (2007). Social dominance in childhood and adolescence: Why social competence and aggression may go hand in hand. In P. H. Hawley, T. D. Little, \&, P. C. Rodkin (Eds.). Aggression and adaptation: The bright side to bad behavior (pp. 1-30). Mahwah: Lawrence Erlbaum Associates.

Hawley, P. H., Little, T. D., y Rodkin, P. C. (2007). Aggression and adaptation: The bright side to bad behavior. Mahwah: Lawrence Erlbaum Associates.

Olthof, T., Goossens, F. A., Vermande, M. M., Aleva, E. A., y van der Meulen, M. (2011). Bullying as strategic behavior: Relations with desired and acquired dominance in the peer group. Journal of School Psychology, 49(3), 339-359. doi: 10.1016/j.jsp.2011.03.003

Prinstein, M. J., y Cillessen, A. H. (2003). Forms and Functions of Adolescent Peer Aggression Associated with High Levels of Peer Status. Merrill-Palmer Quarterly, 49(3), 310-342. doi: $10.1353 / \mathrm{mpq} .2003 .0015$

Schwartz, D., Lansford, J. E., Dodge, K. A., Pettit, G. S., y Bates, J. E. (2015). Peer victimization during middle childhood as a lead indicator of internalizing problems and diagnostic outcomes in late adolescence. Journal of Clinical Child \& Adolescent Psychology, 44(3), 393-404. doi: 10.1080/15374416.2014.881293

Zeman, J., Shipman, K., y Suveg, C. (2002). Anger and sadness regulation: Predictions to internalizing and externalizing symptoms in children. Journal of Clinical Child and Adolescent Psychology, 31(3), 393398. doi: 10.1207/S15374424JCCP3103_11 